\title{
On the identification of time-varying systems in Reproducing Kernel Hilbert Spaces
}

\author{
Gianluigi Pillonetto \\ Control and Dynamic Systems Group, Department of Information Engineering, Via Gradenigo, 6/B - \\ 35131 Padova (Italy), Ph.: +39 0498277607 Fax: +39 0498277699 e-mail: giapi@dei.unipd.it \\ Keywords: Bayes estimation; Tikhonov regularization; Gaussian processes; nonparametric \\ identification; time-varying parameters; compactness in infinite dimensional spaces;
}

\section{Introduction}

During recent years much attention has been devoted to the problem of identification of time-varying systems. To cite some examples, in $[9,17]$ Kalman filter based algorithms for estimation of time-varying parameters are presented and stability and convergence results are derived. A discussion about performance of recursive least squares identification and related adaptive control can be found in $[5,6]$ while in [19] identification of time-varying systems is investigated in the framework of information-based complexity. Neural networks and Markov chain Monte Carlo based identification strategies are proposed in $[20,10]$. A more recent topic includes also the study of the so called linear parameter-varying models, originally introduced in [13], where the system coefficients are rational functions of the parameters. Applications of such models to robust gain scheduling problems can be found in $[14,1]$. In [11] an instance of the problem with state measurements and one parameter is shown to be equivalent to a linear regression while in [3] the identification problem is solved in terms of input/output and parameter trajectory data.

While all the papers mentioned above are concerned with discrete-time models, this paper considers a class of linear state space systems with parameters evolving according to a continuous-time Gauss Markov process. We also assume that such stochastic process admits a state-space representation and that the parameter trajectory is not accessible to direct measurements. Our problem is to reconstruct such trajectory from a finite set of nonlinearly related output data. The novelty of the paper is first to show how this continuous-time identification problem can be embedded under the framework of Tikhonov regularization and reproducing kernel Hilbert space (RKHS) theory [2, 18]. Next, we exploit such theoretical connections to derive a novel identification algorithm which may exhibit significant computational advantages with respect to those procedures that rely upon discretizations of the temporal axis. The current work can also be seen as an extension to state-space models and general RKHSs of that presented in [4].

Recent papers in control literature have studied function estimation problems from the perspective of regularization by assuming a linear relationship between the unknown map and the measurements, see e.g. [8, 12, 15, 16]. In this work we instead consider a nonlinear function estimation problem in an infinitedimensional context and this naturally raises additional and delicate issues. For instance, establishing existence of the optimal trajectory of the time-varying parameters is far from trivial. To overcome these difficulties, the first part of our work will be devoted to the development of a new compactness result related to RKHSs embedded in spaces of continuous functions. Recent work on this subject has lead to results that show pre-compactness of unit balls of RKHSs in the sup-norm (uniform) topology, see e.g. Section 5 in Chapter 3 of [7]. In this paper, we will demonstrate that every scalar Gaussian process that admits a state-space 
representation is isometrically isomorphic to a RKHS whose unit ball is compact in the uniform topology. This result is key since it will allow first to derive conditions on the system to be identified which ensure existence of the solution to the problem. Next, it will exploited to design an efficient numerical procedure for system identification. Our algorithm reconstructs the optimal parameter trajectory by generating a sequence of finite-dimensional Tikhonov-type regularization problems whose solutions converge in the sup-norm topology to that of the original infinite-dimensional problem. In particular, the compactness result guarantees that an accurate approximation of the continuoustime trajectory can be captured by subspaces whose dimension may turn out to be small with respect to the size of observed data. This may thus render the proposed numerical scheme extremely efficient.

\section{REFERENCES}

[1] P. Apkarian and P. Gahinet. A convex characterization of gain scheduled h1 controllers. IEEE Trans. on Automatic Control, pages 853-863, 1995.

[2] N. Aronszajn. Theory of reproducing kernels. Transactions of the American Mathematical Society, 68:337-404, 1950.

[3] B. Bamieh and L. Giarr. Identification of linear parameter varying models. International Journal of Robust and Nonlinear Control, 2002.

[4] B.M. Bell and G. Pillonetto. Estimating parameters and stochastic functions of one variable using nonlinear measurements models. Inverse Problems, 20(3):627-646, 2004.

[5] S. Bittanti and M. C. Campi. Bounded error identification of time-varying parameters by rls techniques. IEEE Trans. on Automatic Control, AC39:1106-1110, 1994.

[6] M.C. Campi. Exponentially weighted least squares identification of time-varying systems with white dusturbances. IEEE Trans. on Signal Processing, 42:2906-2914, 1994.

[7] F. Cucker and S. Smale. On the mathematical foundations of learning. Bulletin of the American mathematical society, 39:1-49, 2001.

[8] T. Evgeniou, M. Pontil, and T. Poggio. A unified framework for regularization networks and support vector machines. CBCL Paper 171, Massachusetts Institute of Technology, Cambridge, MA, March 1999.
[9] L. Guo. Estimating time-varying parameters by the kalman filter based algorithm: Stability and convergence. IEEE Trans. on Automatic Control, 35:1461-1471, 1990.

[10] Chung ki Min. A gibbs sampling approach to estimation and prediction of time-varyingparameter models. Computational Statistics and Data Analysis, 27:171-194, 1998.

[11] R. Ravikanth M. Nemani and B. Bamieh. Identification of linear parametrically varying systems. In Proc. of the 34th IEEE Control and Decision Conference, New Orleans, 1995.

[12] G. De Nicolao, G. Sparacino, and C. Cobelli. Nonparametric input estimation in physiological systems: problems, methods and case studies. Automatica, 33:851-870, 1997.

[13] A. Packard. Gain scheduling via linear fractional transformations. System and Control Letters, 22:79-92, 1994.

[14] J. Shamma. Linearization and gain scheduling. Control Handbook, ED. W. Levine, pages 388-396, 1996.

[15] S. Sun, M.B. Egerstedt, and C.F. Martin. Control theoretic smoothing splines. IEEE Transactions on Automatic Control, 45:2271-2279, 2000.

[16] S. Takahashi, B.K. Gosh, and C.F. Martin. Boundary location using control theoretic splines. Transactions of the Society of Instrument and Control Engineers, 38:293298, 2002.

[17] Y.Z. Tsypkin and M.V. Bondarenko. An optimal algorithm for identification of rapidly time-varying systems. IEEE Trans. on Automatic Control, 37:237-239, 1992.

[18] A.N. Tychonov and V.Y. Arsenin. Solutions of Ill-Posed Problems. Washington, D.C.: Winston/Wiley, 1977.

[19] Le Yi Wang. Persistent identification of timevarying systems. IEEE Trans. on Automatic Control, 42:66-82, 1997.

[20] L. Yingwei, N. Sundararajan, and P. Saratchandran. Identification of timevarying nonlinear systems using minimal radial basis function neural networks. Control Theory and Applications, IEE Proceedings, 144:202-208, 1997. 